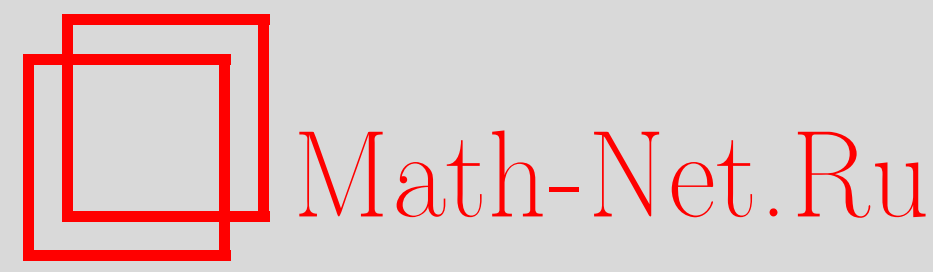

О. Н. Попов, О модулях над кольцом многочленов, получаемых из представлений конечномерных ассоциативных алгебр. II. Случай несовершенного поля, Матем. сб., 2004, том 195, номер 9, 75-84

DOI: https://doi.org/10.4213/sm846

Использование Общероссийского математического портала Math-Net.Ru подразумевает, что вы прочитали и согласны с пользовательским соглашением

http://www.mathnet.ru/rus/agreement

Параметры загрузки:

IP: 54.210 .77 .194

26 апреля 2023 г., 11:02:39 
УДК $512.715 / .717+512.552 .22$

\author{
О.Н. Попов
}

\title{
О модулях над кольцом многочленов, получаемых из представлений конечномерных ассоциативных алгебр. II. Случай несовершенного поля
}

\begin{abstract}
Полученные ранее результаты автора о конструкции коэн-маколеевых модулей над кольцом многочленов, возникшей при исследовании уравнений КошиФуэтэ и обобщенной им с кватернионов на произвольные конечномерные ассоциативные алгебры, переносятся на случай алгебр над несовершенньм полем. А именно показьвается, что для максимально центральных алгебр (введенных Адзумая) получающиеся модули являются коэн-маколеевыми, эта конструкция обладает другими хорошими свойствами и этот класс нельзя расширить. Проделанные в случае совершенного поля вычисления различных инвариантов получающихся модулей остаются в силе.

Библиография: 11 названий.
\end{abstract}

В работе [1] мы рассматривали следующую конструкцию модулей над кольцом многочленов. Пусть $A$ - конечномерная ассоциативная алгебра с 1 над полем $\mathbb{k}$ с базисом $e_{1}, \ldots, e_{d}$, а $\rho: A \rightarrow M_{n}(\mathbb{k})$ - ее матричное представление, отвечающее $A$-модулю $M, \operatorname{dim}_{\mathbb{k}} M=n$. Зададим натуральное $l>1$ и рассмотрим кольцо многочленов $R=\mathbb{k}\left[x_{11}, \ldots, x_{d l}\right]$ и модуль $F_{l}(M)$ над ним, являюшийся фактормодулем свободного $R$-модуля $R^{n}$ по подмодулю, порожденному столбцами матриц представления обших элементов алгебры-матриц $\operatorname{Id}_{j}=\sum_{i} \rho\left(e_{i}\right) x_{i j}, j=1, \ldots, l$.

В [1] исследовался вопрос, когда получаюшиеся модули над кольцом многочленов коэн-маколеевы (сокращенно - CM; это значит, что проективная размерность этих модулей равна высоте их аннулятора в кольце многочленов; сводку требуемых свойств см. в [1; п. 0.1], подробное изложение - в [2]). Было показано [1; теорема 1 , следствие 1], что если алгебра $A$ имеет вид $A=\bigoplus_{i} S_{i} \otimes_{Z\left(S_{i}\right)} K_{i}$, где $S_{i}-$ простые алгебры над $\mathbb{k}, Z\left(S_{i}\right)$ - их центры, а $K_{i}$-конечномерные коммутативные алгебры над соответствуюшими $Z\left(S_{i}\right)$, то все неразложимые $A$-модули переходят в коэн-маколеевы $R$-модули, а если при этом $\operatorname{dim}_{Z\left(S_{i}\right)} S_{i}=n^{2}$ для всех $i$, то и все $A$-модули переходят в коэн-маколеевы. Также было показано, что для таких алгебр $F_{l}(\cdot)$ - точный функтор.

В [1; теорема 2] показано, что над совершенным полем $\mathbb{k}$ указанное выше достаточное условие коэн-маколеевости всех модулей $F_{l}(M)$ является и необходимым и что для точности функтора $F_{l}(\cdot)$ это условие также является необходимым. Затем ставился вопрос, верно ли это для условия коэн-маколеевости образов неразложимых модулей. В работе [3] был дан положительный ответ на этот вопрос.

Работа выполнена при частичной поддержке Российского фонда фундаментальных исследований (грант № 02-01-00468). 
В настоящей работе мы переносим результаты работы [1] на случай несовершенного поля, т.е. доказываем теорему 1 для более широкого (что мы показываем на примере) класса максимально центральных алгебр (введенного Адзумая в [4], [5], см. определение в п. 0.2 настоящей работы), а затем показываем, что коэнмаколеевость образов всех модулей или точность функтора $F_{l}(\cdot)$ влекут принадлежность алгебры этому классу.

В [1] также ставилась задача исследовать свойства алгебры, над которой имеется модуль $M$ с нулевьм аннулятором и с коэн-маколеевьм $F_{l}(M)$ (для некоторого $l>1$ ). Частичные результаты в этом направлении (предложение 2 далее) приводятся и используются по ходу доказательства.

Приступим к точной формулировке результатов.

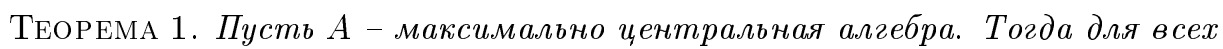
$l>1 F_{l}(\cdot)$ - точный вполне строгий функтор, который переводит неразложимые конечномерные $A$-модули в коэн-маколеевы. Если при этом все простые факторь $A$ имеют одинаковую размерность над своими центрами, то функтор переводит все $A$-модули в коэн-маколеевь. Найденные в [1; теорема 3] численные инварианты получающихся коэн-маколеевых модулей будут такими жсе и в әтом случае.

Tеорема 2. Пусть для некоторого $l>1$ функтор $F_{l}(\cdot)$ точен или же переводит все конечномерные $A$-модули в коэн-маколеевы. Тогда $A-$ максимально чентральная алгебра, причем во втором случае все ее простые факторы имеют одинаковую размерность над своими центрами.

Параграф 0 содержит предварительные сведения, а следующие параграфы посвящены доказательству теорем с соответствуюшими номерами. Обе теоремы доказываются сведением к случаю алгебраически замкнутого поля, который для теоремы 1 разобран в [1].

Автор хотел бы поблагодарить своего научного руководителя Е. С. Голода за эту посильную, но нетривиальную задачу.

\section{§ 0. Предварительные сведения}

0.1. Обозначения и леммы о конечномерных алгебрах. Следуюшие известные леммы несколько раз будут использованы далее.

Мы будем обозначать центр конечномерной алгебры $B$ через $Z(B)$, радикал $\operatorname{rad} B$, инверсно изоморфную алгебру (т.е. то же векторное пространство с умножением $\left.\left(b_{1}, b_{2}\right) \mapsto b_{2} b_{1}\right)$ через $B^{0}$.

Лемма 0.1. Пусть все простые факторы алгебры А имеют размерность $n^{2}$ над своими центрами. Тогда то же верно для алгебры $\bar{A}$, полученной из $A$ переходом к алгебрачческому замыканию основного поля.

ДокАЗАТЕЛЬСТво. Так как при расширении основного поля нильрадикал переходит в нильрадикал, то можно считать алгебру $A$ полупростой, а рассматривая слагаемые по отдельности - простой. Пусть $\overline{\mathbb{k}}$ - алгебраическое замыкание $\mathbb{k}$. Тогда

$$
A \otimes_{\mathbb{k}} \overline{\mathbb{k}} / \operatorname{rad} \bar{A}=A \otimes_{Z(A)}\left(\left(Z(A) \otimes_{\mathbb{k}} \overline{\mathbb{k}}\right) / \operatorname{rad}\left(Z(A) \otimes_{\mathbb{k}} \overline{\mathbb{k}}\right)\right),
$$


так как тензорное произведение центральной простой алгебры на полупростую полупросто [6; теоремы 68.1 и 71.10]. Но второй множитель в этой формуле коммутативная полупростая алгебра над $\overline{\mathbb{k}}$, т.е. сумма нескольких экземпляров $\overline{\mathbb{k}}$, а $A \otimes_{Z(A)} \overline{\mathbb{k}}-$ простая алгебра той же размерности над своим центром [6; теоремa 68.1].

Лемма 0.2. Пусть $A$-конечномерная ассоциативная алгебра с 1 над алгебраически замкнутым полем $\mathbb{k}$, все простье факторы которой имеют размерность $n^{2}$ над свочми центрами. Тогда $A=M_{n}(K)$ для некоторой алгебpbi $K$.

ДокАЗАТЕльство. Так как поле алгебраически замкнуто, то полупростой фактор $A$ есть сумма алгебр $M_{n}(\mathbb{k})$ в некотором числе. По теореме ВеддербернаМальцева [6; теорема 72.19] он вкладывается в $A$ как подалгебра, содержащая единицу $A$ (cp. [1; замечание 0.4.6 и доказательство предложения 2.6, шаг 1]), и если вложить $M_{n}(\mathbb{k})$ в него диагонально, то она тоже вложится в $A$ как подалгебра, содержащая единицу $A$. Так как центр $M_{n}(\mathbb{k})$ есть $\mathbb{k}$, то (ср. [1; замечание 0.4.6 и доказательство предложения 2.6, шаг 2]) можно применить теорему 4.4.2 из [7; гл. IV]. Эта теорема утверждает, что если $S \subset A$ - конечномерная простая подалгебра $L$-алгебры $A$, содержащая единицу алгебры $A$, и $Z(S)=L$, то $A=S \otimes_{Z(S)} K$, где $K$ - централизатор $S$ в $A$. Таким образом, $A=M_{n}(\mathbb{k}) \otimes_{\mathbb{k}} K=$ $M_{n}(K)$.

\section{2. Максимально центральные алгебры.}

ОПРЕДЕЛЕНИЕ [4; $§ 2]$. Конечномерная ассоциативная алгебра $A$ над $\mathbb{k}$ с единицей называется максимально центральной алгеброй, если $A$ - прямая сумма алгебр $A_{i}$, факторы которых по радикалу просты и $\operatorname{dim}_{\mathbb{k}} A_{i} \leqslant t_{i}^{2} \operatorname{dim}_{\mathbb{k}} Z\left(A_{i}\right)$, где $t_{i}^{2}$ - это ранг $A_{i} / \operatorname{rad} A_{i}$ над своим центром и на самом деле имеет место равенство.

В [5] это определение обобщается на случай алгебр над гензелевым кольцом, являюшихся над ним свободными модулями конечного ранга, но мы ограничимся конечномерньми алгебрами над полем. Это определение допускает много равносильных переформулировок (в частности, основные результаты этой работы можно интерпретировать как такую переформулировку), большей частью приведенных в [4], [5].

ПРЕДЛОЖЕНИЕ 1. Следующие условия на конечномерную ассоциативную алгебру А с 1 над полем $\mathbb{k}$ эквивалентни:

1) $А$ - максимально центральная алгебра;

2) при проекции $A$ на любую ее факторалгебру $B$ чентр А сюрвективно отображсается на иентр $B$;

3) алгебра $\bar{A}$, полученная из $A$ переходом $\kappa$ алгебраическому замыканию основного поля, - прямая сумма матричных алгебр над своими чентрами;

4) алгебра $A \otimes_{\mathbb{k}} L$, где $L$ - произвольное расширение поля $\mathbb{k}$, максимально иентральна;

5) $A$ - прямая сумма таких алгебр $A_{i}$ с иентрами $Z_{i}$, ито $A_{i}-$ свободнье $Z_{i}$-модули и $\operatorname{End}_{Z_{i}} A_{i}=A_{i} \otimes Z_{i} A_{i}^{0}$ 
6) A - алгебра Адзумая над своим иентром Z, т.е. [8; гл. VI, §1, определение 1.5] такой проективный $Z$-модуль, что для любого простого идеала $\mathfrak{p} \subset Z \quad A \otimes_{Z} k(\mathfrak{p})$ - чентральная простая алгебра над $k(\mathfrak{p})$.

Если основное поле совериенно, то әти условия эквивалентны следующему:

7) $A=\bigoplus_{i} S_{i} \otimes_{Z\left(S_{i}\right)} K_{i}$, где $S_{i}$ - простые алгебры над $\mathbb{k}$, а $K_{i}-$ конечномерные коммутативные алгебры над соответствующими $Z\left(S_{i}\right)$ ( $A$ - “линейная комбинация простых алгебр с коммутативными коэффициентами”).

ДоказАтельство. Равносильность условий 1$), 3)$ и 4$)$ доказана в $[4 ; \S 2$, теорема 2 и следствие из нее]. Равносильность условий 1) и 5) доказана в [5; п. 4].

Равносильность условий 5) и 6) следует из теоремы 15 работы [5]. Эта теорема утверждает, что $Z_{i}$-алгебра $A_{i}$, являюшаяся свободным $Z_{i}$-модулем, где $Z_{i}$ коммутативное кольцо, обладает свойством, требуемым от так обозначенной алгебры в условии 5), если и только если для любого максимального идеала $\mathfrak{p} \subset Z_{i}$ $A_{i} / \mathfrak{p} A_{i}$ - центральная простая алгебра над $Z_{i} / \mathfrak{p}$. Центр алгебры $A$ - коммутативное артиново кольцо, которое разлагается в сумму локальных артиновых колец, и тогда те же идемпотенты задают разложение $A$ в прямую сумму алгебр с локальньми центрами, так что слагаемые неразложимы. Условие 6) локально по центру, поэтому переносится на каждое слагаемое и, так как конечно порожденный проективный модуль над локальным кольцом свободен, означает, что слагаемые - свободные модули над своими центрами. Условие 5) также переносится на слагаемые, если разложить обе стороны равенства в сумму модулей над слагаемыми центра. А равносильность условий для одного слагаемого утверждается в цитированной вьше теореме 15 из [5].

$3) \Rightarrow 2$ ). Следует из того, что расширение скаляров перестановочно со взятием центра, так как последний задается линейными уравнениями.

$2) \Rightarrow 1$ ). Разложим полупростой фактор $A$ в прямую сумму простых. Это разложение задается некоторым полным семейством центральных ортогональных идемпотентов в полупростом факторе. Так как по условию центр $A$ сюръективно отображается на центр полупростого фактора, то по теореме 24 из [5] (или следствию 7.5 из [9]) это семейство можно поднять до полного семейства ортогональных идемпотентов в центре $A$, которое задает разложение $A$ в прямую сумму алгебр, каждая из которых имеет только один простой фактор и удовлетворяет условию 2).

Для каждого слагаемого $A_{i}$ докажем формулу индукцией по длине Loewy, т.е. по степени нильпотентности нильрадикала. Если нильрадикал нулевой, то $A_{i}$ проста, ее центр - поле и, очевидно, имеет требуемую размерность. Иначе пусть $\left(\operatorname{rad} A_{i}\right)^{n}$ - последняя ненулевая степень радикала. Тогда по предположению индукции центр алгебры $A_{i} /\left(\operatorname{rad} A_{i}\right)^{n}$ имеет требуемую размерность. Центр $A_{i}$ сюръективно отображается на него с ядром, равным $Z\left(A_{i}\right) \cap\left(\operatorname{rad} A_{i}\right)^{n}$, и размерность $A_{i}$ над $\mathbb{k}$ на $\operatorname{dim}_{\mathbb{k}}\left(\operatorname{rad} A_{i}\right)^{n}$ больше, чем у факторалгебры, так что достаточно показать, что $\operatorname{dim}_{\mathbb{k}} Z\left(A_{i}\right) \cap\left(\operatorname{rad} A_{i}\right)^{n}=\operatorname{dim}_{\mathbb{k}}\left(\operatorname{rad} A_{i}\right)^{n} / \operatorname{dim}_{Z\left(S_{i}\right)} S_{i}$, где $S_{i}=A_{i} / \mathrm{rad} A_{i}$. Интересуюший нас $\left(\operatorname{rad} A_{i}\right)^{n}$ - это бимодуль над $A_{i}$, т.е. модуль над $A_{i} \otimes_{\mathbb{k}} A_{i}^{0}$, но заметим, что так как $Z\left(A_{i}\right)$ действует одинаково слева и справа, то на самом деле это модуль над $A_{i} \otimes_{Z\left(A_{i}\right)} A_{i}^{0}$, а поскольку радикал тривиально действует с обеих сторон, а $Z\left(A_{i}\right)$ отображается на центр (полу)простого фактоpa $S_{i}$, то на самом деле это модуль над простой алгеброй $S_{i} \otimes_{Z\left(S_{i}\right)} S_{i}^{0}$, т.е. прямая 
сумма бимодулей, изоморфных $S_{i}$. Пересечение центра $A_{i}$ с нашей степенью радикала - это множество тех элементов, на которые в этой структуре бимодуля $S_{i}$ одинаково действует справа и слева, т.е. прямая сумма центров $S_{i}$. Отсюда следует, что это пересечение имеет требуемую размерность.

Теперь для того чтобы доказать эквивалентность этих условий последнему над совершенным полем, мы воспользуемся обходным путем: если $A$ максимально центральная, то $\bar{A}$ - прямая сумма матричных алгебр над коммутативными, поэтому для нее по [1; теорема 1] функтор $F_{l}(\cdot)$ точен, так что он точен и для $A$, а тогда по $[1$; теорема 2$] A$ имеет требуемьй вид. Наоборот, для $A$ такого вида ясно, что условие 1) выполнено.

ЗАмЕчАниЕ. Над несовершенным полем последнее условие не равносильно предыдущим, как показывает следующий пример.

Пусть $\mathbb{k}=\mathbb{F}_{p}(x)$, где $x$ - независимая переменная. Рассмотрим его чисто несепарабельное расширение $F=\mathbb{F}_{p}(t)=\mathbb{k}[t] /\left(t^{p}-x\right)$. Построим сначала конечномерное центральное тело над $F$, не получающееся при расширении скаляров из такого тела над $\mathbb{k}$.

Пусть $L=\mathbb{F}_{p}(u)=F[u] /\left(u^{p}-u-t\right)$ - циклическое расширение Галуа степени $p$ над $F, \sigma$ - образующий групш Галуа, переводящий $u$ в $u+1$. Тогда согласно [10; $\S 114]$ элементы групшы Брауэра $F$, тривиальные над $L$, представляются как циклические алгебры, т.е. алгебры вида $F\langle\sigma, u\rangle /\left(\sigma^{p}-\alpha, u^{p}-u-t, \sigma u-(u+1) \sigma\right)$ (переменные коммутируют с $F$, но не друг с другом), где $\alpha$ - некоторьй элемент $F^{*}[10$; $\S 94$, п. 4], причем они изоморфны, если и только если соответствующие $\alpha$ отличаются множителем, являющимся нормой некоторого элемента в расширении $L / F$, в частности, циклическая алгебра является полньм матричным кольцом, если и только если $\alpha$ - норма [10; $\S 114$, задача 3]. Рассмотрим циклическую алгебру с $\alpha=t-1$ : это центральная простая алгебра над $F$ размерности $p^{2}$, так что это либо тело, либо матричная алгебра над $F$. Так как $t-1=u^{p}-u-1-$ неприводимьй многочлен в $\mathbb{F}_{p}[u]$, то он не является нормой (т.е. произведением $p$ сопряженных элементов) в расширении $L / F$, значит, наша циклическая алгебра - нетривиальный элемент группы Брауэра, так что она - тело. Если бы это тело получалось из тела над $\mathbb{k}$ расширением скаляров, то тело над $\mathbb{k}$ имело бы ранг $p^{2}$ и задавало бы элемент из $p$-кручения $\operatorname{Br}(\mathbb{k})$ по теореме 4.4 .5 из [7], которая утверждает, что класс тела в группе Брауэра аннулируется квадратным корнем из ранга этого тела. Но при индуцированном расширением скаляров отображении $\operatorname{Br}(\mathbb{k}) \rightarrow \operatorname{Br}(F) p$-кручение отображается в нуль, как мы сейчас покажем. Для этого воспользуемся тем, что группа Брауэра $\operatorname{Br}(\mathbb{k})$ есть объединение своих подгрупп $\operatorname{Br}(\mathbb{k}, K)$, состоящих из алгебр, становящихся изоморфными матричной после тензорного домножения на $K$, где $K$ пробегает конечные расширения Галуа поля $\mathbb{k}$ [10; $\S 113,114]$. А группа $\operatorname{Br}(\mathbb{k}, K)$ изоморфна $H^{2}\left(G, K^{*}\right)$, где $G$ - группа Галуа $K / \mathbb{k}[11 ; \S 6$, п. 8, предложение 11$]$ (по сути это переформулировка описания группы Брауэра через системы факторов). Но согласно $[11 ; \S 6$, п. 8] когомологии групп есть частный случай функтора Ext, поэтому они $\mathbb{Z}$-линейны $[11 ; \S 5$, п. 3, предложение 6], т.е. возведение в степень $p$ в поле $K$ задает умножение на $p$ в $\operatorname{Br}(\mathbb{k}, K)$. Заметим теперь, что гомоморфизм возведения в степень $p$ задает изоморфизм $F$ на $\mathbb{k}$, так что является изоморфизмом и на группах Брауэра, а его композиция с вложением $\mathbb{k} \subset F$ есть возведение в степень $p$ (и продолжается как возведение в степень $p$ на конечные расширения Галуа поля $\mathbb{k})$, так что гомоморфизм $\operatorname{Br}(\mathbb{k}) \rightarrow \operatorname{Br}(F)$ по- 
сле домножения на изоморфизм становится умножением на $p$ и $p$-кручение при нем переходит в нуль.

Итак, требуемое тело построено. Теперь опишем пример максимально центральной алгебры над несовершенным полем, не удовлетворяющей условию 7) предложения 1. Поле $\mathbb{k}$ оставим прежним, положим $B=\mathbb{k}[\widetilde{t}] /\left(\widetilde{t}^{p^{2}}-x^{p}\right)$, а $A=$ $B\langle\sigma, u\rangle /\left(\sigma^{p}-(\widetilde{t}-1), u^{p}-u-\widetilde{t}, \sigma u-(u+1) \sigma\right)$ (здесь $\sigma, u$ коммутируют с $\left.B\right)$. Эта алгебра является циклическим скрещенным произведением в смысле [5; п. 6]. Заметим, что $a=\widetilde{t}^{p}-x-$ нильпотент степени $p$ в $B$ и $B /(a)=F$, так что $A /(a)-$ это наше тело. Также заметим, что $B \subset Z(A)$. Если положить $\sigma>u$ и взять порядок “степенной-лексикографический”, то старшие члены определяющих соотношений $A$ суть $\sigma^{p}, u^{p}, \sigma u$ и не делящиеся на них мономы-это лишш $u^{i} \sigma^{j}, 0 \leqslant i, j<p$, так что $A$ порождается $p^{2}$ мономами как $B$-модуль. Отсюда видно, что условие 1$)$ предложения 1 выполнено, откуда (из того, что неравенство не бывает строгим) следует, что $Z(A)=B$. Если бы $A$ удовлетворяло 7), то, так как фактор $A$ по нильрадикалу - тело, в сумме было бы лишш одно слагаемое, т.е. $A=S \otimes_{Z(S)} K$, где $S$ проста, а $K$ коммутативна и локальна. Тогда $A / \operatorname{rad} A=S \otimes_{Z(S)} K / \operatorname{rad} K$, т.е. наше тело получено из $S$ расширением скаляров с $Z(S)$ до $K / \operatorname{rad} K$. Но центр нашего тела - это $F$ и $[F: \mathbb{k}]=p$, так что $\mathbb{k} \subset Z(S) \subset F$ влечет, что одно из этих включений - равенство. Но наше тело не получается расширением скаляров из тела над $\mathbb{k}$, значит, $Z(S)=F$. Таким образом, $F$ должно вкладываться в $Z(A)=B$ как $\mathbb{k}$-подалгебра с 1 , но оно так не вкладывается: любой прообраз элемента $t \in F$ в $B$ имеет вид $\widetilde{t}+a b$ для некоторого $b \in B$ и $(\widetilde{t}+a b)^{p}=\widetilde{t}^{p}+a^{p} b^{p}=\widetilde{t}^{p}=x+a \neq x$.

0.3. Базисы Грёбнера. Поскольку теория базисов Грёбнера обычно излагается в литературе для идеалов, а не для подмодулей, как она использована в этой работе, мы напомним основные формулировки по [9; гл. 15].

Пусть $R$ - кольцо многочленов над полем $\mathbb{k}$, а $F$ - свободный $R$-модуль с фиксированным базисом $e_{1}, \ldots, e_{s}$. Моном в модуле $F$ - это элемент вида $m e_{i}$, где $m-$ моном в $R$ (т.е. произведение степеней переменных). Порядок на мономах в $F-$ это линейный порядок, для которого если $m_{1}>m_{2}-$ мономы в $F$, а $n \neq 1$ - моном в $S$, то $n m_{1}>n m_{2}>m_{2}$. Любой такой порядок является полньм (непустое подмножество имеет наименьший элемент).

$C$ тариий член элемента $f=\sum a_{i} m_{i} \in F$, где $a_{i} \in \mathbb{k}^{*}$, a $m_{i}$ - различные мономы в $F,-$ это $a_{0} m_{0}$, где $m_{0}$ - наибольший из $m_{i}$, входящих в запись. Если $M \subset F-$ подмодуль, то модуль стариих членов подмодуля $M$ - это подмодуль в $F$, порожденный старшими членами всех элементов $M$. Тогда образы мономов $F$, не лежащих в подмодуле старших членов для $M$, образуют базис $F / M$ как векторного пространства, в частности, если $M$ однородный, то у него и у подмодуля старших членов одинаковая функция Гильберта [9; теорема 15.26].

Пусть некоторьй моном $n$, входящий в элемент $g$ с коэффициентом $a$, делится на старший член $m$ элемента $f$ (домножая $f$ на константу, можно считать, что коэффициент при $m$ в $f$ равен 1$)$. Тогда редукиия $g$ при помощи $f$ - это переход от $g$ к $g-a(n / m) f$. Если ни один моном $g$ не делится на старшие члены элементов $f_{1}, \ldots, f_{k}$, то говорят, что $g$ не редуцируем при помощи $f_{1}, \ldots, f_{k}$. В замечании из п. 0.2 использовался вариант редукции для двусторонних идеалов в кольце некоммутативных многочленов: делимость означала, что $n=n_{1} m n_{2}$, а редукция заменяла $g$ на $g-a n_{1} f n_{2}$. Из полноты порядка следует, что невозможна бесконечная последовательность редукций. 
Набор элементов $g_{1}, \ldots, g_{k}$ модуля $M$ назьвается базисом Грёбнера $M$ для данного порядка, если старшие члены этих элементов порождают модуль старших членов для $M$ (в однородном случае это условие можно проверить по функциям Гильберта). Тогда сами эти элементы порождают $M$.

Если $m_{1}$ и $m_{2}-$ два монома в $F$, в которые входит один и тот же элемент $e_{i}$, то их наименьшее обшее кратное $m$ определяется очевидным образом. Если эти мономы являются старшими членами элементов $f, g \in F$ соответственно, то $S$-форма, соответствующая паре $(f, g),-$ это элемент $\left(m / m_{1}\right) f-\left(m / m_{2}\right) g \in F$.

Критерий Бухбергера [9; теорема 15.8] утверждает, что набор элементов $g_{1}, \ldots, g_{k} \in F$ является базисом Грёбнера порожденного ими подмодуля, если и только если любую S-форму, построенную по паре элементов из этого набора (у которых в старшие члены входит один и тот же базисный элемент $F$ ), можно превратить в нуль последовательностью редукций при помощи элементов этого набора, и тогда если применять к S-фформе редукции при помощи $g_{i}$ в произвольной последовательности и на некотором шаге получится нередуцируемьй при помощи $g_{1}, \ldots, g_{k}$ элемент, то этот элемент будет нулевым.

\section{§1. Доказательство теоремы 1}

Согласно пп. 1)-3) леммы 1.6 из [1] интересующие нас свойства функтора достаточно проверить после расширения поля $\mathbb{k}$ до алгебраического замыкания, но согласно п. 7) предложения 1 в случае алгебраически замкнутого поля наша алгебра имеет вид, для которого все эти свойства функтора доказаны в [1; теорема 1]. Поскольку все рассматривавшиеся в [1] численные инварианты коэн-маколеевых модулей также не меняются при переходе к алгебраическому замыканию поля [1; $§ 3]$, то проведенные там вычисления этих инвариантов остаются в силе.

\section{§ 2. Доказательство теоремы 2}

2.1. Случай точного функтора. Достаточно доказать, что над $\overline{\mathbb{k}}-$ алгебраическим замыканием $\mathbb{k}$ - функтор $F_{l}(\cdot)$ по-прежнему точен. Пусть в обозначениях начала статьи $\widetilde{A}=A \otimes_{\mathbb{k}} R, \widetilde{M}=R \otimes_{\mathbb{k}} M$ - левый $\widetilde{A}$-модуль. Тогда $\widetilde{A}-$ $\widetilde{A}$-бимодуль, так что $F_{l}(A)$ - правый $\widetilde{A}$-модуль и, как показано в начале $\S 1$ работы [1], $F_{l}(M)=F_{l}(A) \otimes_{\widetilde{A}} \widetilde{M}$, что равно $F_{l}(A) \otimes_{\widetilde{A}}\left(\widetilde{A} \otimes_{A} M\right)=F_{l}(A) \otimes_{A} M$, т.е. $F_{l}(\cdot)$ - тензорное умножение на $F_{l}(A)$ над $A$. Но известно, что свойство модуля быть плоским равносильно сохранению точности последовательности конечно порожденных модулей при тензорном домножении на этот модуль $[11 ; \S 4$, п. 6 , теорема 2]. Поэтому точность $F_{l}(\cdot)$ равносильна тому, что $F_{l}(A)$ - плоский правый $A$-модуль. Но это условие сохраняется при расширении скаляров и вообще при индуцировании, что, очевидно, следует из ассоциативности тензорного произведения.

2.2. Случай коэн-маколеевости. Сначала, как в [1; предложение 2.1], покажем, что все простые факторы алгебры $A$ имеют одинаковую размерность над своими центрами. Пусть это не так и $S_{1}$ и $S_{2}$ - два фактора разных размерностей $n_{1}^{2}$ и $n_{2}^{2}$, а $M_{1}$ и $M_{2}$ - простые модули над ними. Тогда при переходе к алгебраическому замыканию поля все простые факторы $S_{i} \otimes_{\mathbb{k}} \overline{\mathbb{k}}$ будут иметь ту же размерность над своими центрами, что и $S_{i}$, так что по [1; лемма 2.8] размерность Крулля $F_{l}\left(M_{i}\right)$ будет равна $\operatorname{dim} R-(l-1) n_{i}-1$. С модулем $F_{l}\left(M_{1} \oplus M_{2}\right)=F_{l}\left(M_{1}\right) \oplus$ 
$F_{l}\left(M_{2}\right)$ будут ассоциированы простые идеалы, минимальные в носителе каждого из $M_{i}$. Так как эти идеалы имеют разную ковысоту (равную размерности Крулля $F_{l}\left(M_{i}\right)$ ), это противоречит теореме несмешанности для коэн-маколеевых модулей [2; Б.1], которая утверждает, что все простые идеалы, ассоциированные с коэнмаколеевьм модулем, имеют одну и ту же ковысоту. Значит, $F_{l}\left(M_{1} \oplus M_{2}\right)$ не коэнмаколеев.

Теперь теорема вытекает из следующего предложения:

ПРЕДЛОЖЕНИЕ 2. Пусть алгебра А такова, что размерности всех ее простых факторов над центрами одинаковы, и для некоторого $l>1$ и некоторого A-модуля $M$ с нулевым аннулятором модуль $F_{l}(M)$ коэн-маколеев. Тогда А максимально центральная.

ДокАЗАТЕльство следует общей схеме доказательств аналогичных утверждений в [1], [3].

Пусть $\overline{\mathbb{k}}$ - алгебраическое замьканиеполя $\mathbb{k}$ и $\bar{A}=A \otimes_{\mathbb{k}} \overline{\mathbb{k}}$. Тогда ясно, что $\bar{A}$ также удовлетворяет условиям предложения, так как тривиальность аннулятора $M$ и коэн-маколеевость $F_{l}(M)$ сохраняются, а по лемме 0.1 размерности простых факторов над центрами также сохраняются.

Пусть размерность простых факторов $A$ над центрами равна $n^{2}$. Тогда по лемме $0.2 \bar{A}=M_{n}(K)$ для некоторой алгебры $K$; так как $\bar{A} / \operatorname{rad} \bar{A}=M_{n}(K / \operatorname{rad} K)$, то все простые факторы $K$ суть $\overline{\mathbb{k}}$.

ЛЕмма 3 (ср. [1; лемма 2.9]). Построенная выше алгебра К удовлетворяет условиям предложения 2.

ДокАЗАтЕЛЬСтво. Известно, что при $\bar{A}=M_{n}(K) \bar{A}$-модуль $M$ имеет вид $\mathscr{M}^{n}$ для некоторого $K$-модуля $\mathscr{M}$. Если у $M$ нулевой аннулятор, то аннулятор $\mathscr{M}$ тоже нулевой. Тогда в (обозначениях 1.7 из [1]) $F_{l}(M)$ есть фактор по столбцам блочной $(n \times l n)$-матрицы $\left(\operatorname{Id}_{i j}^{K}\right)$, где $\operatorname{Id}_{i j}^{K}=\sum_{\alpha} \rho_{\mathscr{M}}\left(f_{\alpha}^{K}\right) x_{\alpha i j}$ - общие элементы для алгебры $K$ от $l n^{2}$ непересекающихся наборов переменных $x_{\alpha i j}, i=1, \ldots, n$, $j=1, \ldots, \ln , \alpha=1, \ldots, \operatorname{dim}_{\overline{\mathbb{k}}} K$. Пусть $f_{1}$ - единица $K$. Рассмотрим последовательность

$\mathbf{x}=\left(\left\{x_{1 i j}-\delta_{i j}\right\}_{i, j=2, \ldots, n},\left\{x_{\alpha i j}\right\}_{\substack{i, j=2, \ldots, n \\ \alpha=2, \ldots, \operatorname{dim}_{\mathbb{k}} K}},\left\{x_{\alpha i j}\right\}_{i \geqslant 2, j \notin[2, n]},\left\{x_{\alpha 1 j}\right\}_{j=2, \ldots, n}\right)$, т.е. такую, что после подстановки этих значений в переменные вьшеуказанная матрица примет вид

$$
\left(\begin{array}{ccccccc}
\operatorname{Id}_{11}^{K} & & \mathbf{0} & & \operatorname{Id}_{1, n+1}^{K} & \ldots & \operatorname{Id}_{1, l n}^{K} \\
0 & 1 & & \mathbf{0} & & & \\
\vdots & & \ddots & & & \mathbf{0} & \\
0 & \mathbf{0} & & 1 & & &
\end{array}\right)
$$

Легко видеть, что фактор по столбцам получившейся матрицы есть $F_{l^{\prime}}(\mathscr{M})$ для $l^{\prime}=(l-1) n+1 \geqslant(2-1) 1+1=2$ и что $\overline{\mathbb{k}}\left[\left(M_{n}(K)\right)^{l}\right] /(\mathbf{x})=\overline{\mathbb{k}}\left[K^{l^{\prime}}\right]$. То есть $F_{l^{\prime}}(\mathscr{M})=F_{l}(M) / \mathbf{x} F_{l}(M)$. Нам надо доказать, что $F_{l^{\prime}}(\mathscr{M})-\mathrm{CM}$, а так как $F_{l}(M)-$ $\mathrm{CM}$ по условию, то по [2; гл. IV, Б.1] достаточно доказать, что $\operatorname{dim} F_{l^{\prime}}(\mathscr{M})=$ $\operatorname{dim} F_{l}(M)-l(\mathbf{x})$. Но $\mathbf{x}-$ регулярная последовательность в $\overline{\mathbb{k}}\left[\left(M_{n}(K)\right)^{l}\right]$ длины $\operatorname{dim} \overline{\mathbb{k}}\left[\left(M_{n}(K)\right)^{l}\right]-\operatorname{dim} \overline{\mathbb{k}}\left[K^{l^{\prime}}\right]$, а так как по лемме 2.8 из [1]

$$
\operatorname{dim} F_{l}(M)=\operatorname{dim} \overline{\mathbb{k}}\left[\left(M_{n}(K)\right)^{l}\right]-l^{\prime}, \quad \operatorname{dim} F_{l^{\prime}}(\mathscr{M})=\operatorname{dim} \overline{\mathbb{k}}\left[K^{l^{\prime}}\right]-l^{\prime},
$$

то размерности такие, как надо, и $F_{l^{\prime}}(\mathscr{M})$ - CM. 
Следующую лемму при желании можно считать обобщением леммы 2.4 из [1].

Лемма 4. Пусть $K$ и $\mathscr{M}-$ как выше. Виберем вложение $K / \operatorname{rad} K=\overline{\mathbb{k}}^{k}$ в $K$ как подалгебры, содержащей $1_{K}$, выберем в $\mathscr{M}$ композиционный ряд

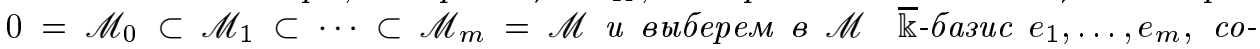
гласованный с этими выборами, т.е. такой, что $\mathscr{M}_{i}=\left\langle e_{1}, \ldots, e_{i}\right\rangle_{\overline{\mathbb{k}}}$ и в ограничении на подалгебру $K / \operatorname{rad} K \overline{\mathbb{K}} e_{i}-$ простые подмодули. Выберем в $K$

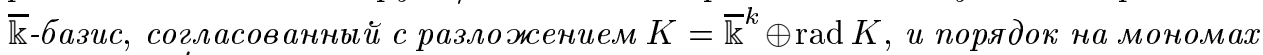
в $R=\overline{\mathbb{k}}\left[K^{l^{\prime}}\right]$. Положим $e_{1}<e_{2}<\cdots<e_{m}$ и зададим на $R^{m}$ порядок “место важнее монома" (т.е. сравнивается номер базисного вектора, а при равенстве - координата). Тогда столбив матриць $\operatorname{Id}_{1}^{K}|\ldots| \operatorname{Id}_{l^{\prime}}^{K}$ образуют базис Грёбнера порожденного ими подмодуля.

ДокАЗАтЕльство. Заметим, что при этих выборах матрица $\operatorname{Id}_{i}^{K}$ является верхнетреугольной, на диагонали стоят переменные, отвечающие простым факторам $K$, а над диагональю - линейные формы от переменных, отвечающих радикалу $K$, так что старшие члены столбцов этой матрицы получаются подстановкой нуля во все переменные, соответствующие радикалу.

Аналогично доказательству предыдущей леммы профакторизуем модуль $F_{l^{\prime}}(\mathscr{M})$ по последовательности переменных, отвечающих базису $\operatorname{rad} K$. Это регулярная последовательность в $\overline{\mathbb{k}}\left[K^{l^{\prime}}\right]$, и фактор этого кольца по ней есть $\overline{\mathbb{k}}\left[(K / \operatorname{rad} K)^{l^{\prime}}\right]$, а фактор $F_{l^{\prime}}^{K}(\mathscr{M})$ по этой последовательности есть $F_{l^{\prime}}^{K / \operatorname{rad} K}(\mathscr{M})$ для ограничения $\mathscr{M}$ на полупростой фактор, вложенный как подалгебра. Так как по лемме 2.8 из [1] размерности Крулля обоих модулей на $l^{\prime}$ менњше размерностей Крулля соответствующих колец, эта последовательность $F_{l^{\prime}}^{K}(\mathscr{M})$-регулярна, так что ряд Гильберта фактора получается из ряда Гильберта $F_{l^{\prime}}^{K}(\mathscr{M})$ умножением на $1-t$ в степени, равной длине последовательности. Значит, если мы тензорно домножим над $\overline{\mathbb{k}} F_{l^{\prime}}^{K / \operatorname{rad} K}(\mathscr{M})$ на кольцо многочленов от переменных, соответствующих радикалу, то получим модуль с той же функцией Гильберта, что и $F_{l^{\prime}}^{K}(\mathscr{M})$. Но мы получим фактор $R^{m}$ по старшим членам столбцов соотношений для $F_{l^{\prime}}^{K}(M)$, откуда и следует, что эти столбцы - базис Грёбнера.

СлЕДСТВИЕ 5. К есть прямая сумма алгебр, над каждой из которых есть только один простой модуль.

ДоКАЗАТЕЛЬство. Сохраним обозначения и выборы базисов из предыдущей леммы. Покажем сначала, что если простые модули $\overline{\mathbb{k}} e_{r}$ и $\overline{\mathbb{k}} e_{s}$ не изоморфны, то матричный элемент $\left(\operatorname{Id}_{i}^{K}\right)_{r s}$ равен 0. При $r>s$ это верно всегда, так что пусть $s>r$. Будем действовать индукцией. Предположим сначала, что при $r<s^{\prime}<s$ $\overline{\mathbb{k}} e_{s^{\prime}}$ не изоморфно $\overline{\mathbb{k}} e_{s}$ как $(K / \operatorname{rad} K)$-модуль. Возьмем S-форму, соответствующую $s$-м столбцам из двух разных $\mathrm{Id}^{K}$, пусть их старшие члены - это $a_{1} e_{s}$ и $a_{2} e_{s}$. Тогда компоненты S-формы с номерами $<s$ суть $a_{1} l_{2}-a_{2} l_{1}$, где $l_{i}-$ линейные формы от переменных, соответствующих радикалу, так что эти компоненты обращаются в нуль, только если $l_{i}=0$, т.е. обрашаются в нуль соответствующие компоненты $s$-х столбцов. Таким образом, если эти компоненты обратились в нуль для всех номеров $\geqslant r$, то $\left(\mathrm{Id}_{i}^{K}\right)_{r s}=0$, а иначе старший член $\mathrm{S}$-формы несет моном от переменных $a_{1}, a_{2}$ и соответствующих радикалу, так что не делится на переменные, соответствующие отличным от $\overline{\mathbb{k}} e_{s}$ простым факторам, и не лежит в подмодуле старших членов столбцов $\mathrm{Id}^{K}$, что дает противоречие с предыдущей леммой. 
Если же между $r$ и $s$ встречаются изоморфные $\overline{\mathbb{k}} e_{s}$ простые модули, то в соответствующих столбцах по предположению индукции $r$-й элемент равен нулю, так что при редукции с их помошью $r$-й элемент S-формы не меняется и в ней не появляются переменные, соответствующие другим простым факторам, так как их нет ни в ней, ни в этих столбцах. Так что когда $\mathrm{S}$-форму нельзя будет редуцировать ими дальше, ее нельзя будет редуцировать никакими столбцами с номерами $\geqslant r$ в любом $\mathrm{Id}^{K}$. Так как она редуцируется к нулю, ее старший член будет выше $r$-й строки, что нам и требовалось.

Итак, утверждение об обрашении матричных элементов в нуль доказано. Отсюда уже следует, что матрицы представления базисных векторов $K / \operatorname{rad} K$ лежат в центре образа $K$ в $\operatorname{End}_{\overline{\mathbb{k}}} \mathscr{M}$, так что эти центральные идемпотенты задают требуемое разложение $K$ в прямую сумму.

Таким образом, $\mathscr{M}$ есть прямая сумма модулей с нулевыми аннуляторами над прямыми слагаемыми $K$, и поскольку конструкция $F_{l}$ над слагаемьм отличается только меньшим числом переменных от конструкции $F_{l}$ над всей алгеброй, то эти слагаемые также удовлетворяют условию предложения 2.

Лемма 6 (ср. [1; лемма 2.10]). Каждое неразложимое слагаемое $K$ коммутативно.

ДоКАЗАТЕльство. Рассмотрим коммутатор двух общих элементов. Тогда его столбцы лежат в подмодуле соотношений и должны редуцироваться к нулю, но они зависят только от переменных, соответствующих нильрадикалу, а старшие члены базиса Грёбнера делятся на переменную, соответствующую полупростому фактору. Поэтому коммутатор нулевой.

Итак, над алгебраически замкнутьм полем предложение доказано. Переход к произвольному - это импликация 3 ) $\Rightarrow$ 1) предложения 1.

\section{Список литературы}

1. Попов О. Н. О модулях над кольцом многочленов, получаемых из представлений конечномерных ассоциативных алгебр // Матем. сб. 2002. Т. 193. № 3. С. 115-134.

2. Серр Ж.-П. Локальная алгебра и теория кратностей // Математика. Сб. пер. 1963. T. 7. № 5. C. 3-93.

3. Попов О. Н. Снова об одной конструкции модулей над кольцом многочленов // УМН. 2003. T. 58. № 2. C. 173-174.

4. Azumaya G., Nakayama T. On absolutely uni-serial algebras // Japan. J. Math. (N.S.). 1948. V. 19. № 4. P. 263-273.

5. Azumaya G. On maximally central algebras // Nagoya Math. J. 1951. V. 2. P. 119-150.

6. Кәртис Ч., Райнер И. Теория представлений конечных групп и ассоциативных алгебр. М.: Наука, 1969.

7. Херстейн И. Некоммутативные кольца. М.: Мир, 1972.

8. Манин Ю. И. Кубические формы. М.: Наука, 1972.

9. Eisenbud D. Commutative algebra with a view toward algebraic geometry. Berlin: Springer-Verlag, 1995. (Grad. Texts in Math. V. 150.)

10. Ван дер Варден Б. Л. Алгебра. М.: Наука, 1979.

11. Бурбаки Н. Алгебра. Гл. Х. М.: Наука, 1987.

Московский государственнњй университет

им. М. В. Ломоносова

Поступила в редакцию

E-mail: popov@mccme.ru

15.10 .2003 